\title{
Modelo para el control de inundaciones utilizando dos embalses en paralelo
}

\section{Model for flood control using two reservoirs in parallel}

\author{
Rafael Fragozo $^{1}$, Víctor Hugo Bedoya ${ }^{2}$, Jesús María López-Lezama ${ }^{3}$ \\ ${ }^{1}$ XM S. A. E. S. P. - Filial de ISA, Colombia. Email: rfragozo@xm.com.co \\ ${ }^{2}$ XM S. A. E. S. P. - Filial de ISA, Colombia. Email: vbedoyar@xm.com.co \\ ${ }^{3}$ Grupo de Manejo Eficiente de la Energía (Gimel), Departamento de Ingeniería Eléctrica, Universidad de Antioquia, Colombia. \\ Orcid: 0000-0002-2369-6173. Email: jmaria.lopez@udea.edu.co
}

Recibido: octubre 18, 2017. Aceptado: febrero 17, 2018. Versión final: marzo 13, 2018.

\begin{abstract}
Resumen
Este artículo presenta un modelo matemático que permite simular el comportamiento de embalses en paralelo, como infraestructura primaria para el control de inundaciones, utilizando el concepto de embalse multipropósito. El modelo se conforma de un conjunto de ecuaciones que representan los flujos de entrada y salida de los embalses y las restricciones asociadas al control de inundaciones. La validación del modelo se realiza utilizando los parámetros técnicos de dos embalses del Sistema Eléctrico Colombiano. Con este desarrollo se ofrece al mercado eléctrico nuevas herramientas orientadas a la toma de decisiones relacionadas con la prevención y el control de inundaciones.
\end{abstract}

Palabras clave: control de inundaciones; embalse multipropósito; fenómeno de La Niña.

\begin{abstract}
This article presents a mathematical model to simulate the behavior of two reservoirs in parallel as primary infrastructure for flood control using the concept of Multipurpose Reservoir. The model is made up of a set of equations that represent the inflows and outflows of reservoirs and constraints associated with flood control. Model validation is performed using the technical parameters of two reservoirs of the Colombian Electric System. With this development, new decision-oriented tools are offered to the electricity market related to flood prevention and control.
\end{abstract}

Keywords: Flood control; multipurpose reservoir; La Niña phenomenon. 


\section{Introducción}

Existen diversos estudios internacionales relacionados con el control de inundaciones y el óptimo funcionamiento de los embalses. En China, por ejemplo, Hongwei y Zezhong, presentaron los beneficios del control de inundaciones a lo largo del río Amarillo. Por su parte, Guohua, Guoli y Shufeng propusieron [2] un esquema de operación de embalses para el control de inundaciones, basado en información en tiempo real y pronósticos para el proyecto de Tres Gargantas. En [3] se plantea un modelo de control de inundaciones para las temporadas de lluvias, aplicando un análisis de confiabilidad y riesgo, basado en los pronósticos hidrológicos también para el proyecto de Tres Gargantas. En Mozambique, se planteó el control de inundaciones utilizando un embalse hidroeléctrico para mitigar el impacto social y reflejando la afectación sobre la generación de energía eléctrica [4]. En Francia, se consideran los elementos de diseño de los diques de protección contra inundaciones [5]. En Vietnam, se propuso para el río Rojo la operación de un embalse basada en varias curvas guía en función del nivel de criticidad [6]. En Malaysia, se propuso la operación de embalses en cascada coordinada por medio de curvas guía, en el cual los volúmenes de los embalses se encuentran relacionados por las curvas [7]. En Canadá, se trató el tema de control de inundaciones de un embalse considerando la afectación de la cuenca aguas abajo de la planta, debido a las descargas según las estaciones climatológicas [8]. En [9] se presenta un estado del conocimiento de los modelos y técnicas de optimización utilizados para la operación de los embalses hidroeléctricos. En [10] se propone un modelo estocástico para el control de inundaciones en Colombia durante el fenómeno de La Niña, utilizando la infraestructura de un embalse hidroeléctrico. En [11] se presenta un estudio de simulación dinámica de inundaciones aplicada a un caso colombiano. Otros estudios relacionados con la hidrología colombiana y el control de embalses son presentados en [12] y [13], respectivamente.

Colombia es un país altamente afectado por los fenómenos climáticos como El Niño y La Niña. Los efectos de estos fenómenos suelen ser de alto impacto en la población, la economía y la infraestructura, especialmente durante las temporadas de lluvias ocasionadas por la presencia del fenómeno de La Niña.

La temporada de lluvias que se vivió en el país entre los años 2010 y 2012 es considerada por los expertos como la más devastadora de los últimos 40 años, y ha sido un punto importante para reflexionar sobre la capacidad de respuesta del país ante los desastres naturales a los cuales se encuentra expuesto. De acuerdo con cifras de la Dirección de Gestión del Riesgo del Ministerio del Interior y de Justicia, la temporada de lluvias del 2010 afectó alrededor del $60 \%$ del territorio colombiano y afectó a más de 2,4 millones de personas [14]. En el año 2017 se activaron nuevamente las alertas ante posibles inundaciones, teniendo en cuenta que el 30,4\% de los embalses de generación del país habían alcanzado su capacidad máxima. Esta situación ha generado preocupación en los pobladores, ganaderos y agricultores ubicados aguas abajo de las centrales de generación [15].

En la figura 1 se ilustra la composición de la generación del Sistema Interconectado Nacional (SIN) [16]. De acuerdo con las cifras publicadas en el Informe Oferta y Generación del Sistema Interconectado Nacional, correspondiente al mes de mayo de 2017, el 65,8 \% de la capacidad efectiva neta del sistema corresponde a plantas despachadas centralmente.

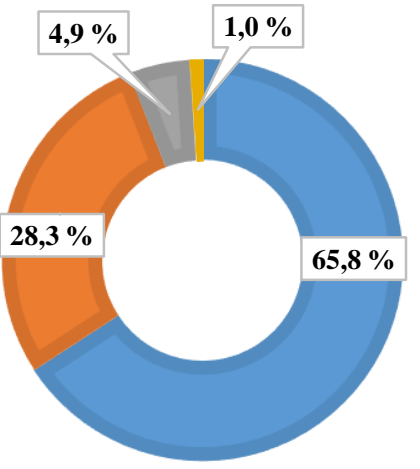

- Centrales Hidroeléctricas

centrales Térmicas

- Plantas Menores

Cogeneradores y Autogeneradores

Figura 1. Porcentajes de Capacidad Efectiva Neta SIN - mayo 2017. Fuente: elaboración propia.

La generación hidráulica en su mayoría es producto de la utilización de los 23 embalses que existen en el país, y que en conjunto suman una capacidad total de almacenamiento de $18647,40 \mathrm{~mm}^{3}$. La coordinación de esta capacidad de almacenamiento, sobre todo de los embalses que fueron construidos tanto para generación de energía eléctrica como para cumplir con fines sociales, juega un papel fundamental a la hora de responder ante los fenómenos climáticos que alteran los ciclos hidrológicos [17]-[19].

En este trabajo se presenta un modelo matemático que permite coordinar la operación de dos embalses en paralelo, con el fin de obtener un balance óptimo entre generación de energía y control de inundaciones, evitando los vertimientos y controlando la zona de inundación aguas abajo, para minimizar de esta forma los riesgos socioambientales asociados con las inundaciones. 


\section{Formulación matemática}

El modelo básico del sistema energético de una planta hidroeléctrica está compuesto por un embalse y sus correspondientes flujos de entrada y salida.

En la figura 2 se ilustra el diagrama de flujos del modelo analizado, en el cual intervienen dos embales. Las variables utilizadas son: flujos aportados por las cuencas aguas arriba de los embalses (Q1 y Q2), flujos de descarga de los embalses con fines de producción de energía eléctrica (G1 y G2), flujos de descarga por vertimiento (V1 y V2), un flujo atribuible a un río externo, el cual no es controlado por los embalses en estudio (Q3), un flujo de desbalance propio de cada embalse (evaporación + filtración) y por último una zona de control de inundaciones, donde se fija un caudal máximo con el fin de proteger una población.

Embalse 1

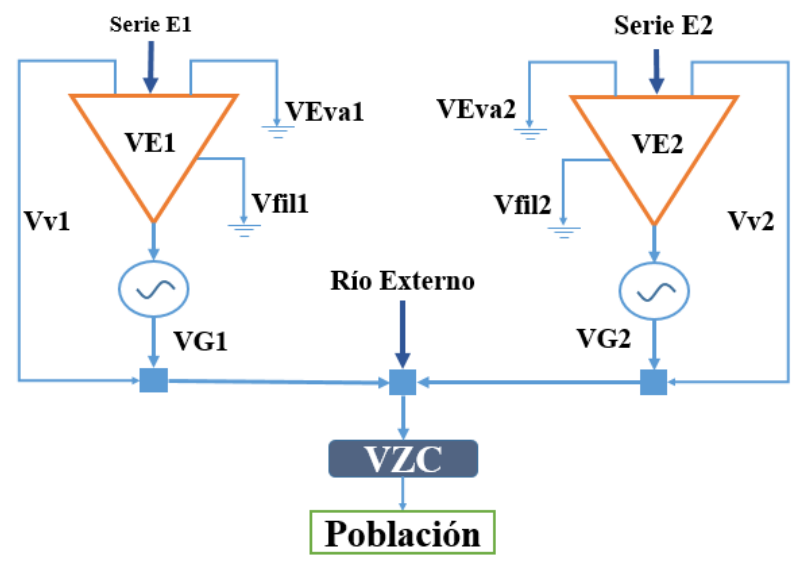

Figura 2. Diagrama de flujos del modelo. Fuente: elaboración propia.

La función objetivo del problema de optimización está dada por la ecuación (1), la cual busca maximizar las utilidades por venta de energía. La función objetivo está compuesta por dos términos, el primero corresponde a la maximización de los ingresos por ventas de energía y el segundo es una penalización por vertimientos. Las restricciones dadas por las ecuaciones (2) y (3) indican los límites mínimos y máximos del volumen de descarga a traves de los ductos de generacion y el volumen de cada embalse, respectivamente. La restricción dada por (4) indica el límite del volumen en la zona de control de inundaciones en cada intervalo de tiempo. La ecuación (5) representa el balance de volúmenes de cada embalse; la ecuacion (6) indica el factor de conversión de generación, y finalmente la ecuación (7) representa el volumen total en la zona de control. En la tabla 1 sedescriben las variables utilizadas en el modelo dado por las ecuaciones (1) a (7).

$$
\begin{aligned}
\operatorname{Maximizar} \sum_{j=1}^{m}( & \left.\sum_{i=1}^{n} P_{i j} * G_{i j}-V_{V_{i j}} * \operatorname{Pen}_{v_{i j}}\right) \\
& -V_{\text {Inu }_{j}} * \operatorname{Pen}_{i n u_{j}}
\end{aligned}
$$

Sujeto a:

$$
\begin{aligned}
& V_{G_{i j} \text { min }} \leq V_{G_{i j}} \leq V_{G_{i j}}{ }_{\text {máx }} \\
& V_{E_{i j} \text { min }} \leq V_{E_{i j}} \leq V_{E_{i j m a ́ x}} \\
& V_{Z C_{j}}-V_{\text {Inu }_{j}} \leq V_{Z C_{\text {máx }}} \\
& V_{E_{i}}=V_{E_{i j-1}}+V_{Q_{i}}-V_{G_{i}}-V_{E F_{i}}-V_{V_{i}} \\
& G_{i}=V_{G_{i}} * F C_{i} \\
& V_{Z C_{j}}=\sum_{i=1}^{n}\left(V_{G_{i j}}+V_{V_{i j}}\right)+V_{Q \text { ext }_{j}}
\end{aligned}
$$

\section{Parámetros para las pruebas}

\subsection{Penalizaciones por inundación}

La figura 2 muestra la zona objetivo para el control de inundaciones localizada aguas abajo de los dos embalses. El modelo de optimización penaliza la función objetivo cuando el flujo que transita por la zona de control de inundación es superior al flujo máximo determinado en el punto de control. Esto es indicado en el primer término de la función objetivo dada por la ecuación (1).

\subsection{Penalizaciones por vertimientos}

El modelo también penaliza la función objetivo cuando se presentan vertimientos. La figura 2 muestra el flujo de vertimiento $V_{V_{i j}}$, el cual es mayor que cero cuando los embalses superan su nivel máximo de almacenamiento. Esta penalización se indica en el segundo término de la función objetivo dada por la ecuación (1).

\subsection{Descripción de las restricciones}

El modelo posee una formulación matemática lineal y sus restricciones son lineales. Las restricciones del modelo corresponden a las características técnicas de los embalses: capacidad mínima y máxima de los embalses, capacidad mínima y máxima de los ductos de generación, capacidad máxima y mínima en la zona de control de la inundación.

Para las restricciones de los embales se consideraron características reales de dos embalses existentes en 
Colombia, de acuerdo con lo consignado en el Documento de Parámetros técnicos del SIN [20].

Tabla 1. Descripción de variables contenidas en la función objetivo y restricciones

\begin{tabular}{|c|c|c|}
\hline Variable & Unidades & Descripción \\
\hline$P_{i j}$ & COP/MWh & $\begin{array}{l}\text { Precio promedio diario de } \\
\text { generación para el embalse } i \text { en el } \\
\text { periodo } j \text {. }\end{array}$ \\
\hline$G_{i j}$ & MWh & $\begin{array}{l}\text { Energía generada a través del } \\
\text { embalse } i \text { en el periodo } j .\end{array}$ \\
\hline$V_{V_{i j}}$ & $\mathrm{Mm}^{3}$ & $\begin{array}{l}\text { Volumen de descarga a través del } \\
\text { vertedero del embalse } i \text { en el } \\
\text { periodo } j \text {. }\end{array}$ \\
\hline $\operatorname{Pen}_{v_{j}}$ & $\mathrm{COP} / \mathrm{Mm}^{3}$ & $\begin{array}{l}\text { Penalización por vertimiento para el } \\
\text { embalse } i \text { en el periodo } j \text {. }\end{array}$ \\
\hline$V_{I n u_{j}}$ & $\mathrm{Mm}^{3}$ & $\begin{array}{l}\text { Volumen de agua por encima del } \\
\text { volumen máximo en la zona de } \\
\text { control de inundación en el periodo } \\
j \text {. }\end{array}$ \\
\hline Pen $_{i n u_{j}}$ & $\mathrm{COP} / \mathrm{Mm}^{3}$ & $\begin{array}{l}\text { Penalización inundación en la zona } \\
\text { de control en el periodo } j \text {. }\end{array}$ \\
\hline$V_{G_{i j}}$ & $\mathrm{Mm}^{3}$ & $\begin{array}{l}\text { Volumen de descarga a través de los } \\
\text { ductos de generación de energía } \\
\text { eléctrica del embalse } i \text { en el periodo } \\
j \text {. }\end{array}$ \\
\hline$V_{G_{i j} j_{m i n}}$ & $\mathrm{Mm}^{3}$ & $\begin{array}{l}\text { Volumen mínimo de descarga a } \\
\text { través de los ductos de generación } \\
\text { de energía eléctrica del embalse } i \text { en } \\
\text { el periodo } j \text {. }\end{array}$ \\
\hline$V_{G_{i j}}{ }_{m a ́ x}$ & $\mathrm{Mm}^{3}$ & $\begin{array}{l}\text { Volumen mínimo de descarga a } \\
\text { través de los ductos de generación } \\
\text { de energía eléctrica del embalse } i \text { en } \\
\text { el periodo } j \text {. }\end{array}$ \\
\hline$V_{E_{i j}}$ & $\mathrm{Mm}^{3}$ & $\begin{array}{l}\text { Volumen del embalse } i \text { en el periodo } \\
j \text {. }\end{array}$ \\
\hline$V_{E_{i \min }}$ & $\mathrm{Mm}^{3}$ & Volumen mínimo del embalse $i$. \\
\hline$V_{E_{i m a ́ x}}$ & $\mathrm{Mm}^{3}$ & Volumen máximo del embalse $i$. \\
\hline$V_{Z C_{j}}$ & $\mathrm{Mm}^{3}$ & $\begin{array}{l}\text { Volumen en la zona de control de } \\
\text { inundaciones en el periodo } j \text {. }\end{array}$ \\
\hline$V_{Z C_{\text {máx }}}$ & $\mathrm{Mm}^{3}$ & $\begin{array}{l}\text { Volumen máximo en la zona de } \\
\text { control de inundaciones. }\end{array}$ \\
\hline$V_{E F_{i}}$ & $\mathrm{Mm}^{3}$ & $\begin{array}{l}\text { Volumen de desbalance compuesto } \\
\text { por la filtración más la evaporación } \\
\text { para el embalse } i \text {. }\end{array}$ \\
\hline$V_{Q i}$ & $\mathrm{Mm}^{3}$ & $\begin{array}{l}\text { Volumen de las cuentas que } \\
\text { ingresan al embalse } i \text { en el periodo } \\
j \text {. }\end{array}$ \\
\hline$F C_{i}$ & $\begin{array}{l}\mathrm{MWh} / \\
\mathrm{Mm}^{3}\end{array}$ & Factor de conversión del embalse $i$. \\
\hline$V_{Q e x t_{j}}$ & $\mathrm{Mm}^{3}$ & $\begin{array}{l}\text { Volumen atribuible a un río externo, } \\
\text { el cual no es controlado por los } \\
\text { embalses en estudio. }\end{array}$ \\
\hline
\end{tabular}

\subsection{Series hidrológicas}

Las simulaciones se realizaron considerando diferentes escenarios de hidrología (baja y alta), tomando como información básica las series hidrológicas históricas de algunos embalses del sistema eléctrico colombiano. Para cada escenario de hidrología se realizaron pruebas al modelo considerando penalizaciones y sin considerar las penalizaciones por vertimiento y por inundación en la zona de control.

Para dar validez al modelo, se tomaron como datos de entrada la Información Hidrológica Oficial del SIN, reportadas por los agentes generadores ante el Consejo Nacional de Operación (CNO) en cumplimiento de la Resolución CREG 071 de 2006.

\subsection{Herramienta de simulación}

Las simulaciones fueron realizadas utilizando la herramienta IBM ILOG CPLEX Optimization Studio. El lenguaje de optimización utilizado es OPL (Optimization Programming Language) y como solucionador se utilizó el optimizador ILOG CPLEX [21].

\section{Resultados}

\subsection{Simulaciones considerando escenarios de hidrología baja}

En la figura 3 se muestra el comportamiento de la energía generada a través de los embalses en GWh/día para el escenario de hidrología baja. Se observa que al activar las penalizaciones por vertimiento y por inundación en la zona de control la energía entregada por el Generador 2 se mantiene estable, lo que disminuye la generación en los casos en los cuales el precio de la energía es bajo (días 7 y 18). El comportamiento de la generación afecta de manera directa los niveles del embalse, como se verifica en la figura 4, en la cual se evidencia la disminución de los embalses, debido a la curva constante de generación de energía, cumpliendo con la restricción en la zona de control como se presenta en la figura 5. 


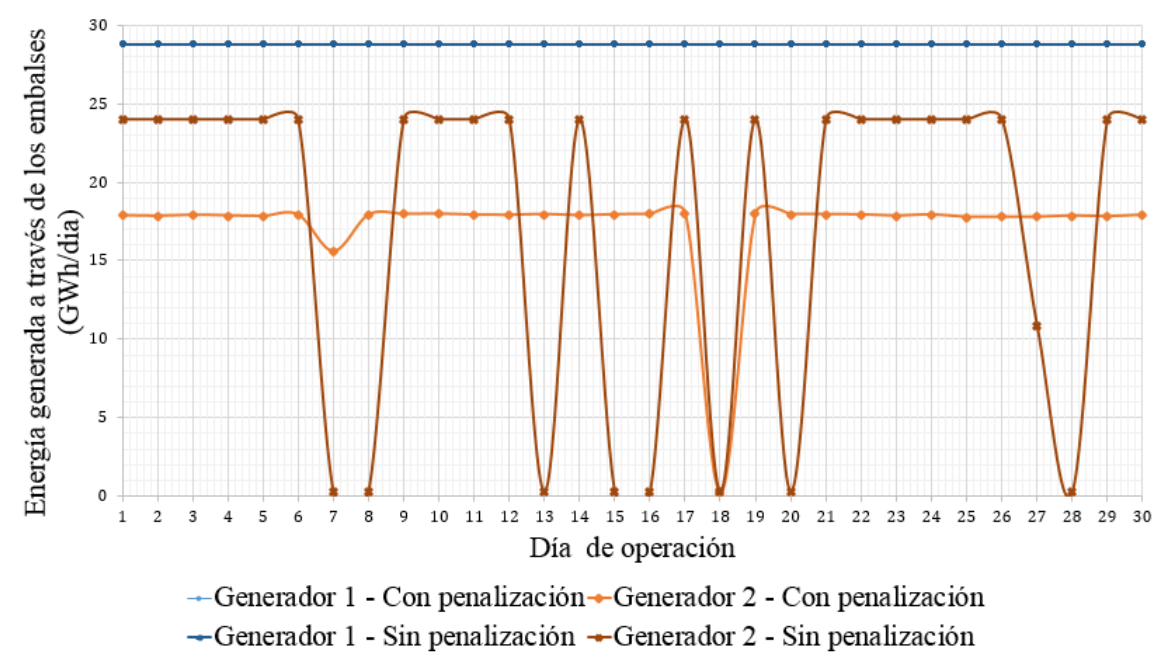

Figura 3. Energía generada a través de los embalses (GWh/día). Fuente: elaboración propia.

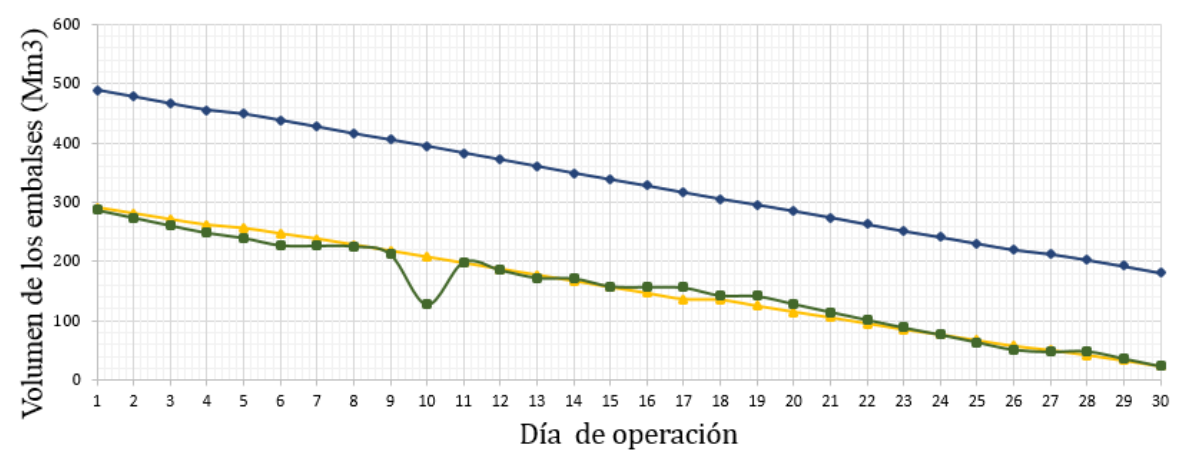

$\rightarrow$ Embalse 1 - Con penalización- $\leadsto$-Embalse 2 - Con penalización

$\rightarrow$ Embalse 1 - Sin penalización $\rightarrow$-Embalse 2 - Sin penalización

Figura 4. Volúmenes de los Embalses (Mm3). Fuente: elaboración propia.

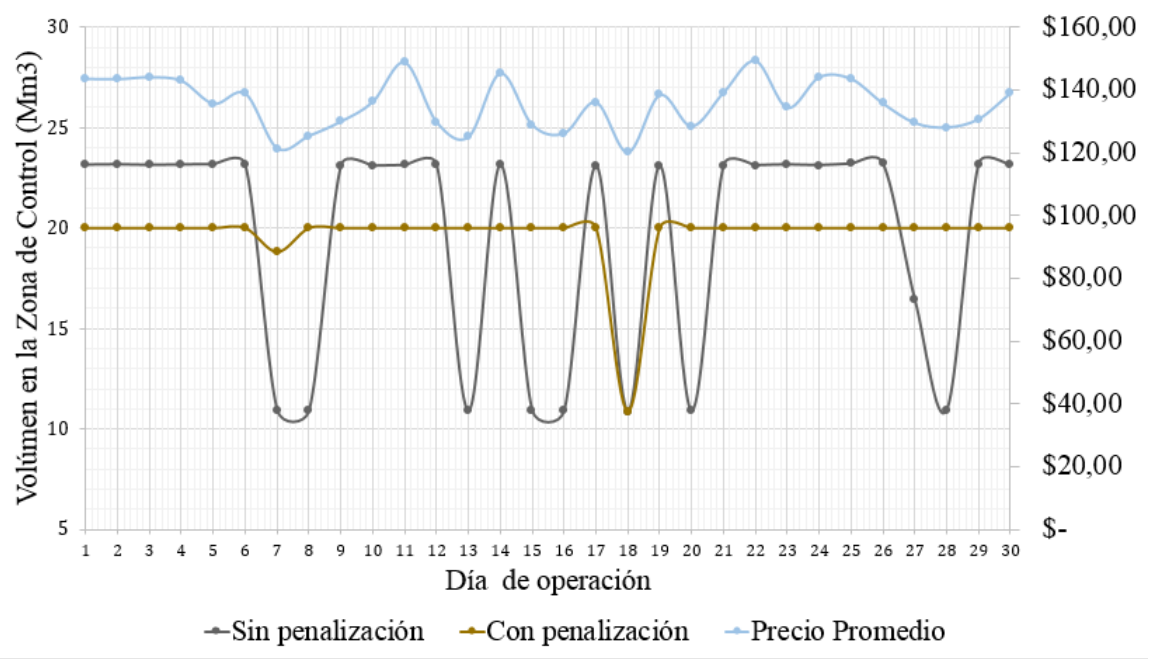

Figura 5. Volumen en la Zona de Control $\left(\mathrm{mm}^{3}\right)$. Fuente: elaboración propia. 


\subsection{Simulaciones hidrología alta}

En la figura 6 se muestra el comportamiento de la energía generada en $\mathrm{GWh} /$ día para el escenario de hidrología alta. Se observa que al activar las penalizaciones por vertimiento y por inundación en la zona de control la energía generada a través de los generadores disminuye, especialmente en el Generador 2, que posee una capacidad de embalse inferior a la del Generador 1. La disminución en los valores de generación se puede verificar en la figura 7 , en la cual se evidencia una curva creciente en el nivel de los embalses para poder cumplir con la restricción en la zona de control, como se presenta en la figura 8 .

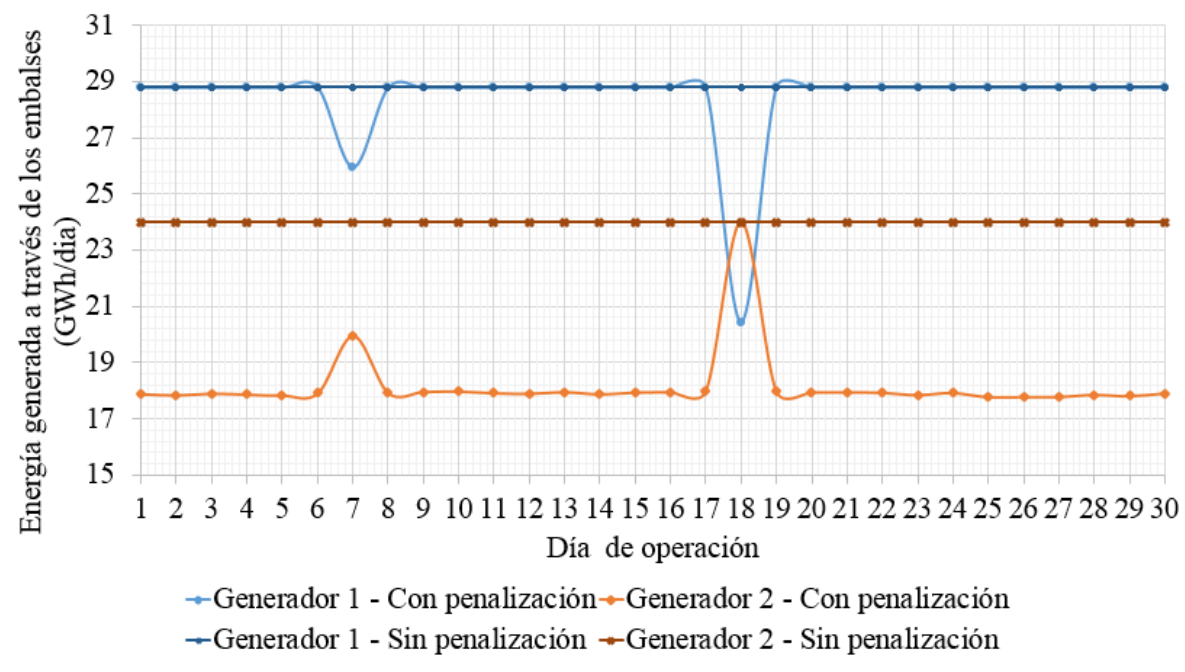

Figura 6. Energía generada a través de los embalses (GWh/día). Fuente: elaboración propia.

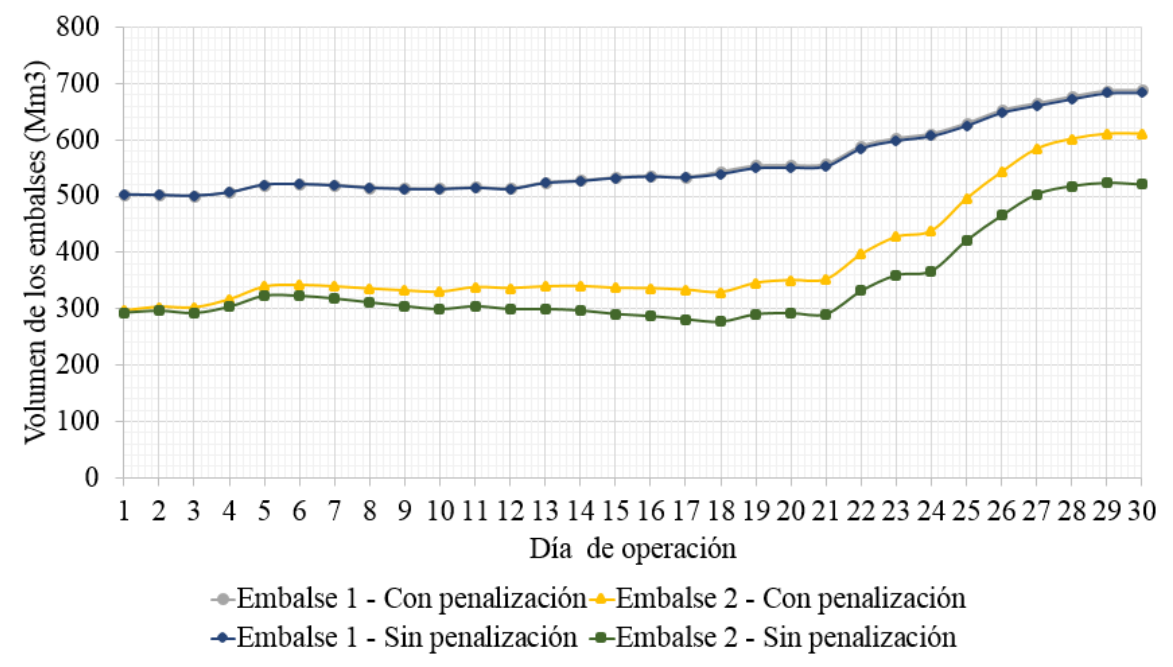

Figura 7. Volúmenes de los Embalses $\left(\mathrm{mm}^{3}\right)$. Fuente: elaboración propia. 


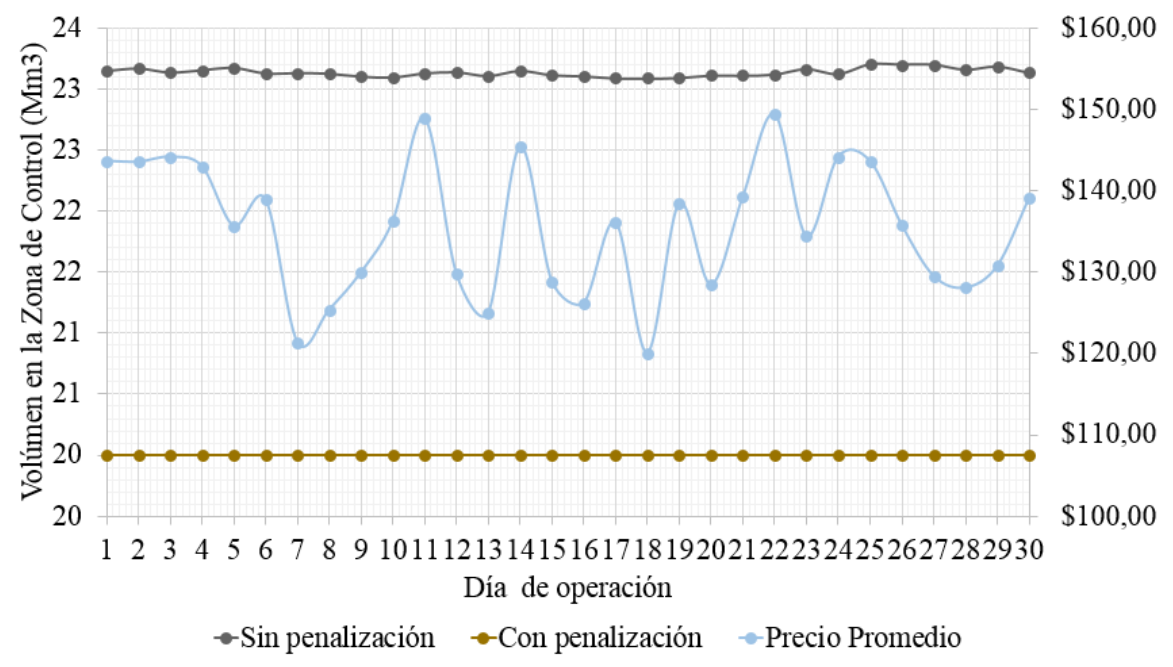

Figura 8. Volumen en la Zona de Control (mm3). Fuente: elaboración propia.

\section{Conclusiones y recomendaciones}

Las ecuaciones lineales planteadas reflejan la realidad de las plantas de generación inmersas en un mercado de energía, cuya función objetivo es la maximización de utilidades bajo los lineamientos del marco regulatorio del país. En el modelo propuesto, los lineamientos están dados por las restricciones técnicas de los embalses y por las penalizaciones para el control de inundaciones en una zona de control.

La confiabilidad en la zona de control de inundaciones aumenta significativamente cuando se incluye en la función objetivo la penalización por inundación. Por esta razón, la combinación de restricciones dada por las características de los embalses y el monitoreo del flujo que se va a controlar se convierten en un factor determinante a la hora de operar de manera coordinada las centrales hidroeléctricas orientadas al control de inundaciones.

El modelo propuesto busca mantener las condiciones actuales del mercado de energía, lo que maximiza las utilidades de las centrales hidroeléctricas por concepto de las ventas de energía, al tiempo que garantiza una alta confiabilidad en la zona de control de inundaciones al considerar penalizaciones por operar por fuera de las características de los embalses, penalizaciones por vertimientos y penalizaciones por inundación en la zona de control.

Este trabajo presenta resultados preliminares de un proyecto mayor cuya esencia es desarrollar modelos hidrometeorológicos con capacidad de predecir y brindar herramientas operativas de cara al fenómeno de La Niña en Colombia. Estos modelos deben ser usados con el ánimo de mitigar los efectos negativos en la población, economía e infraestructura, sin causar mayores impactos en la remuneración de las empresas operadoras de los embalses hidroeléctricos.

\section{Agradecimientos}

Los autores agradecen a la Universidad de Antioquia (UdeA) por el apoyo del proyecto "Sostenibilidad 20162017" para el desarrollo de este trabajo.

\section{Referencias}

[1] H. Du y Z. Zhang, «Longyangxia and Liujiaxia Reservoirs Ecology Flood Compensation Benefits», IEEE 2012 World Autom. Congr., pp. 1-5, 2012.

[2] L. Guohua, W. Guoli, y X. Shufeng, «A Multireservoir Flood Operating System Based on Fuzzy Messages», 2009 Sixth Int. Conf. Fuzzy Syst. Knowl. Discov., pp. 22-28, 2009.

[3] X. Dong, J. Liu, y. Li, H. Bo, Y X. Deng, «Dynamic Application and Risk Analysis of Flood Control Water Level to the Three Gorges Reservoir by Utilizing MidTerm Inflow Forecasts», Power Energy Eng. Conf. (APPEEC), 2010 Asia-Pacific, pp. 1-5, 2010

[4] R. Beilfuss, «Modelling trade-offs between hydropower generation and environmental flow scenarios: a case study of the Lower Zambezi River Basin, Mozambique», Int. J. River Basin Manag., vol. 8, n. ${ }^{\circ}$ 3-4, pp. 331-347, 2010.

[5] D. Goutx, P. Meriaux, y R. Tourment, «Éléments de conception hydraulique des déversoirs des endiguements 
de protection contre les inondations», Ingénieries, vol. Spécial 20, pp. 169-178, 2005.

[6] L. Le Ngo, «Optimising reservoir operation. A case study of the Hoa Binh reservoir, Vietnam», Technical University of Denmark, 2006.

[7] T. Asfaw, K. Yusof, y A. Hashim, «Parameters Estimation and Rule Curve Development of Cascade Hydropower Reservoirs», Natl. Postgrad. Conf. (NPC), $2011,2011$.

[8] C. Fortier, A. Assani, M. Mesfioui, y A. Roy, «Comparison of The Interannual And Interdecadal Variability of Heavy Flood Characteristics Upstream And Downstream From Dams In Inversed Hydrologic Regime: Case Study Of Matawin River (Québec, Canada)», River Res. Appl., vol. 27, n. ${ }^{\circ}$ 10, pp. 12771289, 2011.

[9] J. W. Labadie, «Reservoir System Optimization Models», Water Resour. Updat., vol. 108, pp. 83-110, 1998.

[10]V. H. Bedoya y J. López, «Optimal Flood Control with a Hydroelectric Reservoir: a Colombian Case Study», Transm. Distrib. Conf. Expo. - Lat. Am. (PES T\&D-LA), 2014 IEEE PES, 2014.

[11]S. F. Rodríguez-Corso, "Simulación dinámica de inundaciones asumiendo un estado crítico de máxima escorrentía, bajo cinco periodos de retorno, en la Quebrada la Viergen del Municipio de San José de Miranda-Santander," Rev. UIS Ing., vol. 17, no. 1, pp. 251-270, 2018.

DOI: https://doi.org/10.18273/revuin.v17n1-2018025

[12]D. M. Rey-Valencia, J. del C. Zambrano- Nájera, "Estudio de la respuesa hidrológica en la cuenca urbana de montaña San Luis-Palogrande," Rev. UIS Ing., vol. 17, no. 1, pp. 115-126, 2018.

Doi: https://doi.org/10.18273/revuin.v17n1-2018011

[13] J. C. García-Arredondo, J. M. Ramírez-Scarpetta, "Concepción de un simulador para el entrenamiento de operadores de centrales hidroeléctricas y desarrollo del arranque de un generador," Rev. UIS Ing., vol. 16, no. 2, pp. 105-118, 2017.

Doi: https://doi.org/10.18273/revuin.v16n2-2017010

[14]Organización Panamericana de la Salud, «El fenómeno de La Niña hizo estragos en Colombia», Disasters: Preparedness and Mitigation in the Americas, pp. 6-7, 2011.
[15]Economía y Negocios El Tiempo, «El 30,4\% de los embalses del país está al tope, según firma XM», 15 de mayo 2017, 2017. [En línea]. Disponible en: http://www.eltiempo.com/economia/sectores/embalsesen-colombia-estan-llenos-por-temporada-de-lluvias88476.

[16] XM S.A. E.S.P., XM S.A. E.S.P., «Informe de Oferta y Generación: mayo de 2017», Informe de Oferta y Generación: mayo de 2017, 2017. [En línea]. Disponible en: http://www.xm.com.co/Paginas/Informes.aspx.

[17]D. Raje And P. P. Mujumdar, "Reservoir performance under uncertainty in hydrologic impacts of climate change," Adv. Water Resour., vol. 33, no. 3, pp. 312-326, Mar. 2010.

[18]M. Minville, F. Brissette, y R. Leconte, «Impacts and uncertainty of climate change on water resource management of the Peribonka River system (Canada)», J. water Resour. Plan. Manag., vol. 136, n. ${ }^{\circ} 3$, pp. 376$385,2010$.

[19]L. Guohua, W. Guoli, y X. Shufeng, «A Multireservoir Flood Operating System Based on Fuzzy Messages», 2009 Sixth Int. Conf. Fuzzy Syst. Knowl. Discov., pp. 22-28, 2009.

[20]XM S.A. E.S.P., "Parámetros embalses del SIN," Parámetros técnicos del SIN, 2017. [En línea]. Disponible en : http://www.xm.com.co.

[21]IBM-ILOGIC, «IBM ILOG CPLEX Optimization Studio», IBM ILOG CPLEX Optimization Studio, 2017. [En línea]. Disponible en: https://www.ibm.com/eses/marketplace/ibm-ilog-cplex. 